\title{
Faster Coreset Construction for Projective Clustering via Low-Rank Approximation
}

\author{
Rameshwar Pratap ${ }^{1 \star}$ and Sandeep Sen ${ }^{2}$ \\ 1 Wipro Technologies, Bangalore, India \\ rameshwar .pratap@gmail.com \\ 2 IIT Delhi, India \\ ssen@cse.iitd.ernet.in
}

\begin{abstract}
In this work, we present a randomized coreset construction for projective clustering, which involves computing a set of $k$ closest $j$-dimensional linear (affine) subspaces of a given set of $n$ vectors in $d$ dimensions. Let $A \in \mathbb{R}^{n \times d}$ be an input matrix. An earlier deterministic coreset construction of Feldman et. al. [10] relied on computing the SVD of $A$. The best known algorithms for SVD require $\min \left\{n d^{2}, n^{2} d\right\}$ time, which may not be feasible for large values of $n$ and $d$. We present a coreset construction by projecting the matrix $A$ on some orthonormal vectors that closely approximate the right singular vectors of $A$. As a consequence, when the values of $k$ and $j$ are small, we are able to achieve a faster algorithm, as compared to [10, while maintaining almost the same approximation. We also benefit in terms of space as well as exploit the sparsity of the input dataset. Another advantage of our approach is that it can be constructed in a streaming setting quite efficiently.
\end{abstract}

\section{Introduction}

Succinct representation of Big data - Coreset: Recent years have witnessed a dramatic increase in our ability to collect data from various sources. This data flood has surpassed our ability to understand, analyse and process them. Big data is a new terminology that has become quite popular in identifying such datasets that are difficult to analyse with the current available technologies. One possible approach to manage such large volume of datasets is to keep a succinct summary of the datasets such that it approximately preserves the required properties of the original datasets. This notion was initially formalised by Agrawal et al. 1, and they coined the term coreset for such summaries. Intuitively, a coreset can be considered as a semantic compression of the input. For example: in the case of clustering, a coreset is a weighted subset of the data such that the cost of a clustering algorithm evaluated on the coreset closely approximates to the corresponding cost on the entire dataset. Consider a set $Q$ (possibly of infinite size) of query shapes (for example: subspaces, set of points, set of lines etc.), then for every shape $q \in Q$, the sum of distances from $q$ to the input points, and the sum of distances from $q$ to the points in the coreset, is approximately the same. If the

\footnotetext{
* This work done when author was affiliated with TCS Innovation Labs.
} 
query set belongs to some particular candidate query set, then such coreset is called as a weak coreset [15]; and if the coreset approximates the distances from all possible (potentially infinite) query shapes, then it is called as strong coreset. Coresets are a practical and flexible tool which require no or minimal assumption on the data. Although the analysis techniques for coreset construction are a bit involved, and require tools from computational geometry and linear algebra, the resulting coreset construction algorithms are easy to implement. Another important property of coresets is that they can be constructed in a streaming and distributed setting quite efficiently. This is due to the fact that unions of coresets are coresets, and coresets of coresets are also coresets 12. Also, using these properties it is possible to construct coresets in a tree-wise fashion which can be parallelized in a Map-Reduce style [10].

Coreset constructions have been studied extensively for various data analysis tasks. There are usually two steps involved in the coreset construction - dimensionality reduction, and cardinality reduction. The dimension reduction step of the coreset construction includes projecting points in a low dimension space such that the original geometry of points is also preserved in the low dimension. These projection techniques includes SVD decomposition, random projections, row/column subset selections, or any combinations of these (see [10/5]). The cardinality reduction step includes contracting the input size via sampling or other geometric analysis approach on the reduced dimension instance of the input. We refer readers to survey articles of Jeff M. Phillips [16] and Agarwal et al. 22.

In this work, we focus on the dimension reduction step of coreset construction for the projective clustering problem. In the paragraph below, we discuss the motivation behind the projective clustering problem.

Projective clustering: Clustering is one of the most popular techniques for analyzing large data, and is widely used in many areas such as classification, unsupervised learning, data mining, indexing, pattern recognition. Many popular clustering algorithms such as $k$-means, BIRCH [19], DBSCAN [6] are full dimensional - they give equal importance to all the dimensions while computing the distance between two points. These clustering algorithms works well in low dimensional datasets, however, due to the "curse of dimensionality" such algorithms scale poorly in high dimensions. Moreover, in high dimensional datasets a full dimensional distance might not be appropriate as farthest neighbour of a point is expected to be roughly as close as its nearest neighbour [14. These problems are often handled via methods such as Principal component analysis (PCA) or Johnson-Lindenstrauss lemma by finding a low dimensional representation of the data obtained by projecting all points on a subspace so that the information loss is minimized. However, projecting all the points in a single low dimensional subspace may not be appropriate when different clusters lie in different subspaces. This motivates the study of projective clustering which involves finding clusters along different subspaces. Projective clustering algorithms have been widely applicable for indexing and pattern discovery in high dimensional datasets. 


\subsection{Our contribution}

With the above motivation we study the dimension reduction step of coreset construction for projective clustering problem. We first briefly describe the subspace and projective clustering problems. In a $j$-subspace clustering problem, given a set of $n, d$ dimensional vectors, denoted by $A \in \mathbb{R}^{n \times d}$, the problem is to find a $j$-dimensional subspace such that it minimizes the sum of squared distances from the rows of $A$, over every $j$-dimensional subspace. Further, in the problem of linear (affine) $(k, j)$-projective clustering, the goal is to find a closed set $\mathcal{C}$ which is the union of $k$ linear (affine) subspaces each of dimension $j$, such that it minimizes the sum of squared distances from the rows of $A$, over every possible choice of $\mathcal{C}$ (see Definitions 819).

Feldman et al. 10 presented a deterministic coreset construction for these clustering problems. Their coreset construction relies on projecting the rows of $A$ on the first few right singular values of $A$. However, the main drawback of their construction is that it requires computing the SVD of $A$ which is expensive for large values of $n$ and $d$. Cohen et al. 5 suggested "projection-costpreserving-sketch" for various clustering problems. Their sketches are essentially the dimensionality reduction step of the coreset construction. Using a low rank approximation of $A$, they suggested a faster coreset construction for the subspace clustering problem. However, it was not clear that how their techniques could be extended for projective clustering problem. In this work, we extend their techniques and obtain a faster dimension reduction for projective clustering, and as a consequence, a faster coreset construction for the projective clustering problem. In Section 3, we first revisit the techniques for subspace clustering problem, and in Section 4 we present our coreset construction for projective clustering problem. We state our main result as follows: (In the following theorem, $\mathbf{n n z}(A)$ denotes the number of non-zero entries of $A$.)

Theorem 1. Let $A \in \mathbb{R}^{n \times d}, \epsilon \in(0,1)$, and $j, k$ be two integers less than $(d-1)$, and $(n-1)$ respectively such that $k(j+1) \leq d-1$. Then there is a randomized algorithm which outputs a matrix $A^{*}$ of rank $O\left(k(j+1) / \epsilon^{2}\right)$ such that for every non-empty closed set $\mathcal{C}$, which is the union of $k$ linear (affine) subspaces each of dimension at most $j$, the following holds w.h.p.

$$
\left|\left(\operatorname{dist}^{2}\left(A^{*}, \mathcal{C}\right)+\Delta^{*}\right)-\operatorname{dist}^{2}(A, \mathcal{C})\right| \leq \epsilon \operatorname{dist}^{2}(A, \mathcal{C}) .
$$

Where, $j^{*}=k(j+1) ; \Delta^{*}=\left\|A-A^{O\left(\frac{j^{*}}{\epsilon^{2}}\right)}\right\|_{F}^{2} ; \operatorname{dist}^{2}(A, \mathcal{C})$ denotes the sum of squared distances from each row of $A$ to its closest point in $\mathcal{C}$; and $A^{O\left(\frac{j^{*}}{\epsilon^{2}}\right)}$ is the best rank $O\left(\frac{j^{*}}{\epsilon^{2}}\right)$ approximation of $A$. The running time of the algorithm is $O\left(\mathbf{n n z}(A) \frac{j^{*}}{\epsilon^{3}}+(n+d) \frac{j^{* 2}}{\epsilon^{6}}\right)$.

Remark 1. We develop our coreset by projecting points on some orthonormal vectors that closely approximate the right singular vectors of $A$, and we obtain them using the algorithm of Sarlós [17. The running time of our algorithm is better than the corresponding deterministic algorithm of [10 when $n \geq d$ and $j^{*}=o(n)$, or, when $n<d$ and $j^{*}=o(d)$, where $j^{*}=k(j+1)$. Further, 
as the coreset construction time depends on the number of non-zero entries of the matrix, our algorithm is substantially faster for sparse data matrices. Please note that one can also use any other low-rank approximation algorithms such as 4] (instead of [17]), which offer multiplicative approximation guarantee. However, for completeness sake we use the bounds of [17, and compare our results with [10].

Remark 2. The term $\Delta^{*}$ is a positive constant, and is sum of squared singular values from $O\left(j^{*} / \epsilon^{2}\right)$ to $d$. We use $A^{*}$ to approximately solve the clustering problem, and add the constant $\Delta^{*}$ in the clustering cost obtained from $A^{*}$, this sum gives a good approximation w.r.t. the cost of clustering on $A$.

Remark 3. An advantage of our coresets is that it can be constructed in the pass efficient streaming model [13, where access to the input is limited to only a constant number of sequential passes. We construct our coreset by projecting the matrix $A$ on orthonormal vectors, that closely approximate the right singular vectors of $A$, our algorithm requires only two passes over the data in order to compute those orthonormal vectors using [17.

\subsection{Related work}

Coreset construction has been studied extensively for the problem of $j$-subspace clustering. However, we will discuss a few of them that are more relevant to our work. Feldman et al. 7] developed a strong coreset whose size is exponential in $d, j$, logarithmic in $n$, and their coreset construction requires $O(n)$ time. Feldman et al. 9] improved their earlier result [7] and developed a coreset of size logarithmic in $n$, linear in $d$, and exponential in $j$. However, the construction requires $O(n d j)$ time. In [8] Feldman and Langberg showed a coreset construction of size polynomial in $j$ and $d$ (independent of $n$ ). Feldman et al. [10] presented a novel coreset construction for subspace and projective clustering. They showed that the sum of squared Euclidean distance from $n$ rows of $A \in \mathbb{R}^{n \times d}$ to any $j$-dimensional subspace can be approximated upto $(1+\epsilon)$ factor, with an additive constant which is the sum of a few last singular values of $A$, by projecting the points on the first $O(j / \epsilon)$ right singular vectors of $A$. Thus, they were able to show the dimension reduction from $d$ to $O(j / \epsilon)$. They also showed $O\left(k(j+1) / \epsilon^{2}\right)$ dimension reduction for $(k, j)$-projective clustering problem. Recently, for $j$-subspace clustering, Cohen et al. 5] improved the construction of [10] using only first $\lceil j / \epsilon\rceil$ right singular vectors, which is an improvement over [10] by a constant factor.

Sariel Har-Peled [11] showed that for projective clustering problem it is not possible to get a strong coreset of size sublinear in $n$, even for a simpler instance such as a family of pair of planes in $\mathbb{R}^{3}$. However, in a restricted setting, where points are on an integer grid, and the largest coordinate of any point is bounded by a polynomial in $n$ and $d$, Varadarajan et al. [18] showed that a sublinear sized coreset construction for projective clustering.

Organization of the paper: In Section 2, we present the necessary notations, definitions and linear algebra background that are used in the various proofs 
in the paper. In Section 3, we revisit the result of [5], and discuss the coreset construction for subspace clustering using their techniques. In Section 4, we extend the result of Section 3 and present the coreset construction for projective clustering problem. We conclude our discussion, and state some open questions in Section 5 .

\section{Preliminaries}

\begin{tabular}{|c|l|}
\hline \multicolumn{2}{|c|}{ Notations } \\
\hline$A=U \Sigma V^{T}$ & $\begin{array}{l}\text { columns of } U, V \text { are orthonormal and called as left and right } \\
\text { singular vectors of } A ;[\Sigma] \text { is a diagonal matrix having the } \\
\text { corresponding singular values }\end{array}$ \\
\hline$A^{(m)}=U \Sigma^{(m)} V^{T}$ & $\begin{array}{l}\Sigma^{(m)} \text { is the diagonal having the } m \text { largest entries of } \Sigma \text {, and } \\
0 \text { otherwise }\end{array}$ \\
\hline$[X]_{d \times j}$ & $\begin{array}{l}j \text { orthonormal columns represent a } j \text {-dimensional subspace } \\
L \text { in } \mathbb{R}^{d}\end{array}$ \\
\hline$\left[X^{\perp}\right]_{d \times(d-j)}$ & $(d-j)$ dimensional subspace $L^{\perp}$ orthogonal to subspace $L$ \\
\hline$\pi_{\mathcal{S}}(A)$ & matrix formed by projecting $A$ on the row span of $\mathcal{S}$ \\
\hline$\pi_{\mathcal{S}, k}(A)$ & $\begin{array}{l}\text { the best rank- } k \text { approximation of } A \text { after projecting its rows } \\
\text { on the row span of } \mathcal{S}\end{array}$ \\
\hline$A^{(k)}$ & the best rank- $k$ approximation of $A$ \\
\hline $\mathbf{n n z}(A)$ & the number of non-zero entries of $A$ \\
\hline
\end{tabular}

Below we present some necessary linear algebra background. We first present some basic properties of Frobenius norm of a matrix. We define SVD (singular value decomposition) of a matrix, and its basic properties. We describe the expression about the distance of a point, and sum of square distances of the rows of matrix - from a subspace and a closed set.

Fact 1 (Frobenius norm and its properties) Let $A \in \mathbb{R}^{n \times d}$, then square of Frobenius norm of $A$ is defined as the sum of the absolute squares of its elements, i.e. $\|A\|_{F}^{2}=\Sigma_{i=1}^{n} \Sigma_{j=1}^{d} a_{i, j}^{2}$. Further, if $\left\{\sigma_{i}\right\}_{i=1}^{d}$ are singular values of $A$, then $\|A\|_{F}^{2}=\Sigma_{i=1}^{d} \sigma_{i}^{2}$. Also, if $\operatorname{tr}(A)$ be the trace of the matrix $A$ then $\|A\|_{F}^{2}=\operatorname{tr}\left(A^{T} A\right)$.

Fact 2 Let $A X$ be the projection of points of $A$ on the $j$-dimensional subspace $L$ represented by an orthonormal matrix $X$. We can also write the projection of the points in the rows of $A$ to $L$ as $A X X^{T}$, these projected points are still $d$-dimensional, but lie within the $j$-dimensional subspace. Further, $\|A X\|_{F}^{2}=$ $\left\|A X X^{T}\right\|_{F}^{2}$.

The Singular Value Decomposition: A matrix $A \in \mathbb{R}^{n \times d}$ of rank at most $r$ can be written due to its SVD decomposition as $A=\Sigma_{i=1}^{r} \sigma_{t} u^{(i)} v^{(i)^{T}}$. Here, $u^{(i)}$ and $v^{(i)}$ are $i$-th orthonormal columns of $U$ and $V$ respectively, and $\sigma_{1} \geq \sigma_{2}, \ldots \sigma_{r} \geq 0$. Also, $u^{(i)^{T}} A=\sigma_{i} v^{(i)^{T}}$, and $A v^{(i)}=\sigma_{i} u^{(i)}$ for $1 \leq i \leq r$. Further, the matrix $A^{(k)}$ that minimizes $\|A-B\|_{F}$ among all matrices $B$ (of rank at most $k$ ) is given by $A^{(k)}=\sum_{i=1}^{k} A v^{(i)} v^{(i)^{T}}$ - i.e. by projecting $A$ on the first $k$ right singular vectors of $A$. 
$l_{2}$ distances to a subspace: Let $L$ be a $j$-dimensional subspace in $\mathbb{R}^{d}$ represented by an orthonormal matrix $X \in \mathbb{R}^{d \times j}$. Then, for a point $p \in \mathbb{R}^{d},\left\|p^{T} X\right\|_{F}^{2}$ is the squares of the length of projections of the point $p$ on the subspace $L$. Similarly, given a matrix $A \in \mathbb{R}^{n \times d},\|A X\|_{F}^{2}$ is the sum of squares of the length of projections of the points (rows) of $A$ on the subspace $L$. Let $L^{\perp}$ be the orthogonal complement of $L$ represented by an orthonormal matrix $X^{\perp} \in \mathbb{R}^{d \times(d-j)}$. Then, $\left\|A X^{\perp}\right\|_{F}^{2}$ is the sum of squares of distances of the points of $A$ from $L$.

$l_{2}$ distance to a closed set: Let $S \in \mathbb{R}^{d}$ be a closed set and $p$ be a point in $\mathbb{R}^{d}$. We define the $l_{2}$ distance between $p$ and $S$ by $\operatorname{dist}^{2}(p, S):=\min _{s \in S} \operatorname{dist}^{2}(p, s)$, i.e., the smallest distance between $p$ and any element $s \in S$. If $S$ consists of union of $k, j$-dimensional subspaces $L_{1}, \ldots, L_{k}$, then $\operatorname{dist}^{2}(p, S)$ denotes the distance from $p$ to the closest set $S$. Similarly, given a matrix $A \in \mathbb{R}^{n \times d}$, $\operatorname{dist}^{2}(A, S):=$ $\sum_{i=1}^{n} \operatorname{dist}^{2}\left(A_{i^{*}}, S\right)$. Here, $A_{i^{*}}$ denotes the $i$ th row of $A$.

Pythagorean theorem: Let $A \in \mathbb{R}^{n \times d}, L$ be a $j$-dimensional subspace in $\mathbb{R}^{d}$ represented by an orthonormal matrix $X \in \mathbb{R}^{d \times j}$, and $L^{\perp}$ be the orthogonal complement of the subspace $L$ represented by an orthonormal matrix $X \in \mathbb{R}^{d \times d-j}$. Then by Pythagorean theorem we have $\|A\|_{F}^{2}=\|A X\|_{F}^{2}+\left\|A X^{\perp}\right\|_{F}^{2}$. Further, if $\mathcal{C}$ is a closed set spanned by $X$, then due to the Pythagorean theorem we have $\operatorname{dist}^{2}(A, \mathcal{C})=\left\|A X^{\perp}\right\|_{F}^{2}+\operatorname{dist}^{2}\left(A X X^{T}, \mathcal{C}\right)$. We will use the following fact in our analysis which hold true due to Pythagorean theorem.

Fact 3 Let $A \in \mathbb{R}^{n \times d}$, and $X \in \mathbb{R}^{d \times j}$ be a matrix having first $j$ right singular vectors of $A$ as columns, then due to the Pythagorean theorem, we have $\left\|A-A X X^{T}\right\|_{F}^{2}=\|A\|_{F}^{2}-\left\|A X X^{T}\right\|_{F}^{2}$.

In the following, we state some facts from elementary linear algebra which are required for deriving the correctness of our result.

Fact 4 For a square matrix $M \in \mathbb{R}^{n \times n}, \operatorname{tr}(M)$ is the sum of all its diagonal entries. Further, for matrices $A \in \mathbb{R}^{n \times d}, B \in \mathbb{R}^{d \times n}$ due to the cyclic property of the $\operatorname{tr}$ function, we have $\operatorname{tr}(A B)=\operatorname{tr}(B A)$. Also for square matrices $M, N \in$ $\mathbb{R}^{n \times n}$, due to the linear property of the $\operatorname{tr}$ function: $\operatorname{tr}(M \pm N)=\operatorname{tr}(M) \pm \operatorname{tr}(N)$.

Fact 5 A symmetric matrix $M \in \mathbb{R}^{n \times n}$ is positive semidefinite if $x^{T} M x>0$ for all $x \in \mathbb{R}^{n}$. A matrix $M$ is positive semidefinite then the following two statements are equivalent:

- there is a real nonsingular matrix $N$ such that $M=N^{T} N$,

- all eigenvalues of $M$ are nonnegative.

Fact 6 Let $A \in \mathbb{R}^{n \times d}$ and $U \Sigma V^{T}$ be the $S V D$ of $A$. Then, the first $j$ columns of $V$ span a subspace that minimizes the sum of squares distances of the vectors in $A$ from all $j$-dimensional subspace, and this sum is $\Sigma_{i=j+1}^{d} \sigma_{i}^{2}$. Thus, for any $j$-dimensional subspace represented by an orthonormal matrix $X$, we have $\left\|A X^{\perp}\right\|_{F}^{2} \geq \Sigma_{i=j+1}^{d} \sigma_{i}^{2}$. 
Fact 7 Let $M \in \mathbb{R}^{d \times l}$ be a matrix. Then, for an orthonormal matrix $X \in \mathbb{R}^{d \times k}$, due to elementary linear algebra we have, $\left\|X X^{T} M\right\|_{F}^{2} \leq\|M\|_{F}^{2}$.

In the following, we state the definitions of subspace and projective clustering.

Definition 8 (Subspace clustering) Let $A \in \mathbb{R}^{n \times d}$ and $j$ be an integer less than $d$. Then, the problem of $j$-subspace clustering is to find a $j$-dimensional subspace $L$ of $\mathbb{R}^{d}$ that minimizes the $\operatorname{dist}^{2}(A, L)$. In other words, the goal is to find a matrix $X^{\perp} \in \mathbb{R}^{d \times(d-j)}$ having orthonormal columns that minimizes $\left\|A X^{\perp}\right\|_{F}^{2}$ over every such possible matrix $X^{\perp}$.

Definition 9 (linear (affine) $(k, j)$-projective clustering) Let $A \in \mathbb{R}^{n \times d}$, $j$ be an integer less than $d$, and $k$ be an integer less than $n$. Then, the problem of linear (affine) $(k, j)$-projective clustering is to find a closed set $\mathcal{C}$, which is the union of $k$ linear (affine) subspaces $\left\{L_{1}, \ldots L_{k}\right\}$ each of dimension at most $j$, such that it minimizes the $\operatorname{dist}^{2}(A, \mathcal{C})$, over every possible choice of $\mathcal{C}$.

Theorem 2 (Low-rank approximation by [17]). Let $A \in \mathbb{R}^{n \times d}$, and $\pi$.(.) denote the projection operators stated in the notation table. If $\epsilon \in(0,1]$ and $\mathcal{S}$ is an $(r \times n)$ Johnson-Lindenstrauss matrix with i.i.d. zero-mean \pm 1 entries and $r=O\left(\frac{m}{\epsilon}\right) \log \frac{1}{\delta}$, then with probability at least $1-\delta$ it holds that

$$
\left\|A-\pi_{\mathcal{S A}, m}(A)\right\|_{F}^{2} \leq(1+\epsilon)\left\|A-A^{(m)}\right\|_{F}^{2} .
$$

Further, computing the singular vectors spanning $\pi_{\mathcal{S} A, m}(A)$ in two passes 3 over the data requires $O\left(\mathbf{n n z}(A) r+(n+d) r^{2}\right)$ time.

For our analysis, we will use a weak triangle inequality which is stated below:

Lemma 10 (Lemma 7.1 of [10]) For any $\varepsilon \in(0,1)$, a closed set $\mathcal{C}$, and two points $p, q \in \mathbb{R}^{d}$, we have

$$
\left|\operatorname{dist}^{2}(p, \mathcal{C})-\operatorname{dist}^{2}(q, \mathcal{C})\right| \leq \frac{12\|p-q\|^{2}}{\varepsilon}+\frac{\varepsilon}{2} \operatorname{dist}^{2}(p, \mathcal{C}) .
$$

\section{$3 \quad$ Faster coreset construction for subspace clustering}

In this section after revisiting the results of Cohen et al. [5], we present a randomized coreset construction for subspace clustering. The deterministic coreset construction of Feldman et al. 10, for subspace clustering problem relies on projecting the input matrix on its first few right singular vectors - projecting the rows of $A$ on first few right singular vectors of $A$ - which requires SVD computation of $A$. Cohen et al. [5] suggested that projecting the rows of $A$ on some orthonormal vectors that closely approximate the right singular vectors of $A$ (obtained via e.g. [17) also satisfies the required properties of coreset w.h.p., and as a consequence, gives a faster coreset construction.

\footnotetext{
${ }^{3}$ Two passes are required as we first multiply $A$ on the right with a JohnsonLindenstrauss matrix $\mathcal{S}$, and then we project the rows of $A$ again onto the row span of $\mathcal{S} A$.
} 
Theorem 3 (Adapted from Theorem 8 of [5]). Let $X \in \mathbb{R}^{d \times j}$ be an orthonormal matrix representing a subspace $L$, let $X^{\perp} \in \mathbb{R}^{d \times(d-j)}$ be the orthonormal matrix representing the orthogonal complement of $L, \epsilon \in(0,1), \delta \in(0,1)$, $m=\left\lceil\frac{j}{\epsilon}\right\rceil, \Delta=\left\|A-A^{(m)}\right\|_{F}^{2}$, and $\tilde{A}$ is a rank $m$ approximation of $A$ satisfying Theorem 2. Then, the following is true with probability at least $1-\delta$ :

$$
0 \leq\left|\left\|\tilde{A} X^{\perp}\right\|_{F}^{2}+\Delta-\left\|A X^{\perp}\right\|_{F}^{2}\right| \leq 2 \epsilon\left\|A X^{\perp}\right\|_{F}^{2} .
$$

Proof. Using a result of Sarlós [17, we get a rank $m$ approximation of $A$. If $\mathcal{S}$ is an $(r \times n)$ JL matrix, where $r=O\left(\left(\frac{m}{\epsilon}+m \log m\right) \log \frac{1}{\delta}\right)$ (see Theorem 2) then the following is true with probability at least $1-\delta$ :

$$
\left\|A-\pi_{\mathcal{S} A, m}(A)\right\|_{F}^{2} \leq(1+\epsilon)\left\|A-A^{(m)}\right\|_{F}^{2} .
$$

Here, $A^{(m)}$ is the best $m$ rank approximation of $A$. Let $R^{\prime}$ be the matrix having the first $m$ right singular vectors of $\pi_{\mathcal{S} A}(A)$, and let we denote $A R^{\prime} R^{\prime T}$ by $\tilde{A}$, then by Equation 1 the following holds true with probability at least $1-\delta$ :

$$
\|A-\tilde{A}\|_{F}^{2} \leq(1+\epsilon)\left\|A-A^{(m)}\right\|_{F}^{2}
$$

In the following we show an upper bound on the following expression:

$$
\begin{aligned}
& \left|\left\|\tilde{A} X^{\perp}\right\|_{F}^{2}+\Delta-\left\|A X^{\perp}\right\|_{F}^{2}\right| \\
& =\left|\|\tilde{A}\|_{F}^{2}-\|\tilde{A} X\|_{F}^{2}+\left\|A-A^{(m)}\right\|_{F}^{2}-\|A\|_{F}^{2}+\|A X\|_{F}^{2}\right| \\
& =\left|\|\tilde{A}\|_{F}^{2}-\|\tilde{A} X\|_{F}^{2}+\|A\|_{F}^{2}-\left\|A^{(m)}\right\|_{F}^{2}-\|A\|_{F}^{2}+\|A X\|_{F}^{2}\right| \\
& =\left|\|\tilde{A}\|_{F}^{2}-\left\|A^{(m)}\right\|_{F}^{2}-\|\tilde{A} X\|_{F}^{2}+\|A X\|_{F}^{2}\right| \\
& \leq\left|\left\|A^{(m)}\right\|_{F}^{2}-\left\|A^{(m)}\right\|_{F}^{2}+\|A X\|_{F}^{2}-\|\tilde{A} X\|_{F}^{2}\right| \\
& =\left|\|A X\|_{F}^{2}-\|\tilde{A} X\|_{F}^{2}\right| \leq 2 \epsilon\left\|A X^{\perp}\right\|_{F}^{2}
\end{aligned}
$$

Equality 3 follows from Pythagorean theorem; Equality 4 follows from Fact 3 , where $A^{(m)}=A V^{\prime} V^{\prime T}$, and $V^{\prime} \in \mathbb{R}^{d \times m}$ having $m$ columns from the first $m$ right singular vectors of $A$; Inequality 5 holds as the value of $\|\tilde{A}\|_{F}^{2}-\left\|A^{(m)}\right\|_{F}^{2}$ can be at most zero, because at the best we can hope to sample the right singular vectors of $A$ as $R^{\prime}$, which maximizes the value of the desired expression; finally Inequality 6 holds from Lemma 11

A proof of the following lemma follows from the analysis of Lemma 5 of [5]. We defer its proof in the appendix.

Lemma 11 (Adapted from Lemma 5 and Theorem 8 of [5]) Let $A \in \mathbb{R}^{n \times d}$, $\tilde{A}$ is a rank $m$ approximation of $A$ satisfying Equation 2 , then

$$
0 \leq\|A X\|_{F}^{2}-\|\tilde{A} X\|_{F}^{2} \leq 2 \epsilon\left\|A X^{\perp}\right\|_{F}^{2}
$$




\section{Faster coreset construction for projective clustering}

In this section, extending the result (Theorem 3) of the previous section, we present a randomized coreset construction for the problem of projective clustering. More precisely, if $L_{1}, \ldots, L_{k}$ be a set of $k$ subspaces each of dimension at most $j$, and let $\mathcal{C}$ be a closed set containing union of them, then our randomized coreset is a matrix of very small rank (independent of $d$ ) and it approximately preserves the distances from every such closed set $\mathcal{C}$, with high probability. Our main contribution is the dimensionality reduction step of the coreset construction, which is presented in Algorithm 1 below.

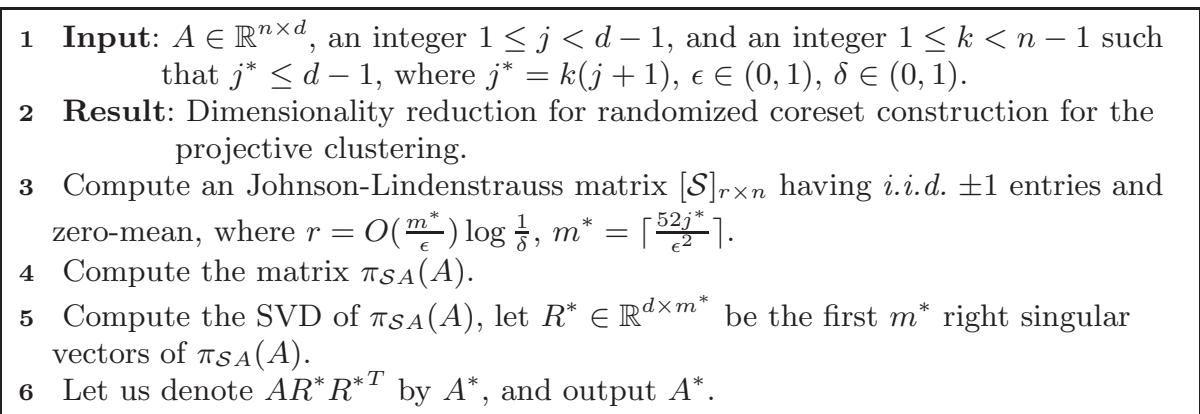

Algorithm 1: Dimensionality reduction for projective clustering.

Proof of Theorem [1 Let $\left[X^{*}\right]_{d \times j^{*}}$ be a matrix with orthonormal columns whose span is $L^{*}$, and let $L^{* \perp}$ be the orthogonal complement of $L^{*}$ spanned by $\left[X^{* \perp}\right]_{d \times\left(d-j^{*}\right)}$. If $\mathcal{C}$ is a closed set spanned by $L^{*}$, then due to the Pythagorean theorem, we have, $\operatorname{dist}^{2}(A, \mathcal{C})=\left\|A X^{* \perp}\right\|_{F}^{2}+\operatorname{dist}^{2}\left(A X^{*} X^{* T}, \mathcal{C}\right)$. Further,

$$
\begin{aligned}
& \left|\left(\operatorname{dist}^{2}\left(A^{*}, \mathcal{C}\right)+\Delta^{*}\right)-\operatorname{dist}^{2}(A, \mathcal{C})\right| \\
& =\left|\left(\left\|A^{*} X^{* \perp}\right\|_{F}^{2}+\operatorname{dist}^{2}\left(A^{*} X^{*} X^{* T}, \mathcal{C}\right)+\Delta^{*}\right)-\left(\left\|A X^{* \perp}\right\|_{F}^{2}+\operatorname{dist}^{2}\left(A X^{*} X^{* T}, \mathcal{C}\right)\right)\right| \\
& \leq \underbrace{\left|\left(\left\|A^{*} X^{* \perp}\right\|_{F}^{2}+\Delta^{*}-\left\|A X^{* \perp}\right\|_{F}^{2}\right)\right|}_{\text {first term }}+\underbrace{\left.\mid\left(\operatorname{dist}^{2}\left(A^{*} X^{*} X^{* T}, \mathcal{C}\right)-\operatorname{dist}^{2}\left(A X^{*} X^{* T}, \mathcal{C}\right)\right)\right) \mid}_{\text {second term }}
\end{aligned}
$$

We have to bound two terms in the above expression. The first term can be upper bounded using a similar analysis as of Theorem 3 which holds true with probability at least $1-\delta$. (In Theorem 3 we replace $j$ by $j^{*}, m$ by $m^{*}, \epsilon$ by $\frac{\epsilon^{2}}{52}$, and $\Delta$ by $\Delta^{*}$.)

$$
||\left|A^{*} X^{* \perp}\right|_{F}^{2}+\Delta^{*}-\left\|\left.A X^{* \perp}\right|_{F} ^{2} \mid \leq \frac{\epsilon^{2}}{26}\right\| A X^{* \perp} \|_{F}^{2}
$$

To bound the second term $\left.\mid \operatorname{dist}^{2}\left(A^{*} X^{*} X^{* T}, \mathcal{C}\right)-\operatorname{dist}^{2}\left(A X^{*} X^{* T}, \mathcal{C}\right)\right) \mid$, we use Lemma 10. For any $\varepsilon \in(0,1)$ and due to Lemma 10, we have

$$
\left.\mid \operatorname{dist}^{2}\left(A^{*} X^{*} X^{* T}, \mathcal{C}\right)-\operatorname{dist}^{2}\left(A X^{*} X^{* T}, \mathcal{C}\right)\right) \mid
$$




$$
\begin{aligned}
& \leq \frac{12}{\varepsilon}\left\|A^{*} X^{*} X^{* T}-A X^{*} X^{* T}\right\|_{F}^{2}+\frac{\varepsilon}{2} \operatorname{dist}^{2}\left(A X^{*} X^{* T}, \mathcal{C}\right) \\
& \leq \frac{12}{\varepsilon}\left(\frac{\epsilon^{2}}{26}\left\|A X^{* \perp}\right\|_{F}^{2}\right)+\frac{\varepsilon}{2} \operatorname{dist}^{2}\left(A X^{*} X^{* T}, \mathcal{C}\right) \\
& \leq \frac{12}{\varepsilon}\left(\frac{\epsilon^{2}}{26}\left\|A X^{* \perp}\right\|_{F}^{2}\right)+\frac{\varepsilon}{2} \operatorname{dist}^{2}(A, \mathcal{C})
\end{aligned}
$$

Inequality 8 holds due to Lemma 12. Thus, we have

$$
\left.\mid \operatorname{dist}^{2}\left(A^{*} X^{*} X^{* T}, \mathcal{C}\right)-\operatorname{dist}^{2}\left(A X^{*} X^{* T}, \mathcal{C}\right)\right) \mid \leq \frac{12}{\varepsilon}\left(\frac{\epsilon^{2}}{26}\left\|A X^{* \perp}\right\|_{F}^{2}\right)+\frac{\varepsilon}{2} \operatorname{dist}^{2}(A, \mathcal{C})
$$

Equation 7 in conjunction with Equation 9, gives us the following:

$$
\begin{aligned}
& \left|\left(\operatorname{dist}^{2}\left(A^{*}, \mathcal{C}\right)+\Delta^{*}\right)-\operatorname{dist}^{2}(A, \mathcal{C})\right| \\
& \leq\left(1+\frac{12}{\varepsilon}\right) \frac{\epsilon^{2}}{26}\left\|A X^{* \perp}\right\|_{F}^{2}+\frac{\varepsilon}{2} \operatorname{dist}^{2}(A, \mathcal{C}) \\
& \leq\left(1+\frac{12}{\varepsilon}\right) \frac{\epsilon^{2}}{26} \operatorname{dist}^{2}(A, \mathcal{C})+\frac{\varepsilon}{2} \operatorname{dist}^{2}(A, \mathcal{C}) \\
& =\left(\frac{\epsilon^{2}}{26}+\frac{12 \epsilon^{2}}{26 \varepsilon}+\frac{\varepsilon}{2}\right) \operatorname{dist}^{2}(A, \mathcal{C}) \\
& =\left(\frac{\epsilon^{2}}{26}+\frac{12 \epsilon}{26}+\frac{\epsilon}{2}\right) \operatorname{dist}^{2}(A, \mathcal{C}) \\
& \leq \epsilon \operatorname{dist}^{2}(A, \mathcal{C})
\end{aligned}
$$

Equality 10 holds by choosing $\varepsilon=\epsilon$, and as $\epsilon^{2} / 26+12 \epsilon / 26<\epsilon / 2$.

A proof of the following lemma is presented in the appendix.

Lemma 12 Let $X^{*} \in \mathbb{R}^{d \times j^{*}}$ be a matrix with orthonormal columns whose span is $L^{*}$, then in Algorithm 1 the following is true with probability at least $1-\delta$

$$
\left\|A^{*} X^{*} X^{* T}-A X^{*} X^{* T}\right\|_{F}^{2} \leq \frac{\epsilon^{2}}{26}\left\|A X^{* \perp}\right\|_{F}^{2} .
$$

Remark 4. Please note that it is sufficient to store the matrix $A R^{*}$ which is of dimension $m^{*}$, where $m^{*}=O\left(k(j+1) / \epsilon^{2}\right)$. However, for the purpose of our analysis, we use the matrix $A R^{*} R^{* T}$ which is of dimension $d$, and rank $m^{*}$. Further, the space that is required to store our coreset is $O\left(n m^{*}+1\right)$ - we need $O\left(n m^{*}\right)$ space to store the matrix $A R^{*}$, and $O(1)$ space to store the term $\Delta^{*}$; on the other hand, the space require to store $A$ is $O(n d)$.

Comparison with coreset construction of [10]: Coreset construction of [10] requires projecting the rows of $A$ on its first $O\left(k(j+1) / \epsilon^{2}\right)$ right singular vectors which gives a matrix of rank $O\left(k(j+1) / \epsilon^{2}\right)$ and it approximately preserves the distance from any closed $\mathcal{C}$. Their construction requires computing SVD of the 
given matrix $A$, which has the run-time complexity of $\min \left\{n^{2} d, n d^{2}\right\}$. In our construction, we showed that it is also sufficient to project the rows of $A$ on $O\left(k(j+1) / \epsilon^{2}\right)$ othronormal vectors that closely approximate the right singular vectors of $A$. We now give an time bound on the running time of Algorithm 1. Time required for execution of line number $3,4,5$ is

$$
O\left(\operatorname{nnz}(A) \frac{m^{*}}{\epsilon}+(n+d)\left(\frac{m^{*}}{\epsilon}\right)^{2}\right)=O\left(\operatorname{nnz}(A) \frac{j^{*}}{\epsilon^{3}}+(n+d) \frac{j^{* 2}}{\epsilon^{6}}\right),
$$

due to [17, where $j^{*}=k(j+1)$. Further, line number 6 requires time - for projecting $A$ on $R^{*}$, which due to an elementary matrix multiplication is $O\left(\mathbf{n n z}(A) m^{*}\right)=$ $O\left(\mathbf{n n z}(A) \frac{j^{*}}{\epsilon^{2}}\right)$. Thus, total running time of Algorithm 1 is

$$
O\left(\mathbf{n n z}(A) \frac{j^{*}}{\epsilon^{3}}+(n+d) \frac{j^{* 2}}{\epsilon^{6}}+\mathbf{n n z}(A) \frac{j^{*}}{\epsilon^{2}}\right)=O\left(\mathbf{n n z}(A) \frac{j^{*}}{\epsilon^{3}}+(n+d) \frac{j^{* 2}}{\epsilon^{6}}\right) .
$$

Clearly, if $n \geq d$ and $j^{*}=o(n)$, or, if $n<d$ and $j^{*}=o(d)$, then our running time is better than that of [10].

As a corollary of Theorem[1, and using the known techniques from [10 1818] on $A^{*}$, we present the cardinality reduction step of coreset construction as follows:

Corollary 13 (Corollary 9.1 of [10]) Let $A \in\{1,2, \ldots, \Lambda\}^{n \times d}$, with $\Lambda \in(n d)^{O(1)}$, $d \in n^{O(1)}$. There is a matrix $\mathcal{Q} \in \mathbb{R}^{l \times d^{\prime}}$ with $l=\operatorname{poly}\left(2^{k j}, \frac{1}{\epsilon}, \log n, \log \Lambda\right)$, $d^{\prime}=O\left(k(j+1) / \epsilon^{2}\right)$; and a weight function associated with the rows of $\mathcal{Q}$, i.e. $w: \mathcal{Q}_{i^{*}} \rightarrow[0, \infty)$ such that for every closed set $\mathcal{C}$, which is the union of $k$ affine $j$-subspaces of $\mathbb{R}^{d}$, the following holds with high probability

$$
(1-\epsilon) \sum_{i=1}^{n} \operatorname{dist}^{2}\left(A_{i^{*}}, \mathcal{C}\right) \leq \Sigma_{i=1}^{l} w\left(\mathcal{Q}_{i^{*}}\right) \operatorname{dist}^{2}\left(\mathcal{Q}_{i^{*}}, \mathcal{C}\right) \leq(1+\epsilon) \sum_{i=1}^{n} \operatorname{dist}^{2}\left(A_{i^{*}}, \mathcal{C}\right) .
$$

As a corollary of Theorem 1, we present randomized coreset result for $k$-mean clustering. However, Theorem 8 of [5] independently offers a tighter dimension reduction bound $\left\lceil\frac{k}{\epsilon}\right\rceil$ for $k$-means.

Corollary 14 Let $A \in \mathbb{R}^{n \times d}, \epsilon \in(0,1)$, and $k$ an integer less than $(d-1)$ and $(n-1)$. Then there is a randomized algorithm which outputs a matrix $A^{\prime}$ of dimension $O\left(k / \epsilon^{2}\right)$ such that for every set of $k$ points $\left\{c_{i}\right\}_{i=1}^{k} \in \mathbb{R}^{d}$ represented as the rows of matrix $C$, the following holds with high probability:

$$
\left|\left(\operatorname{dist}^{2}\left(A^{\prime}, C\right)+\Delta^{\prime}\right)-\operatorname{dist}^{2}(A, C)\right| \leq \epsilon \operatorname{dist}^{2}(A, C) .
$$

The running time of the algorithm is $O\left(\mathbf{n n z}(A) \frac{k}{\epsilon^{3}}+(n+d) \frac{k^{2}}{\epsilon^{6}}\right)$. Where, $\Delta^{\prime}=$ $\left\|A-A^{O\left(k / \epsilon^{2}\right)}\right\|_{F}^{2}$; and $\operatorname{dist}^{2}(A, C)$ denotes the sum of square distances from each row of $A$ to its closest point in $C$.

\section{Conclusion and open problems}

We presented a randomized coreset construction for projective clustering via low rank approximation. We first revisited the result of [5] for the subspace clustering, and then extended their result to construct a randomized coreset for projective clustering. We showed that our construction is significantly faster (when 
the values of $k$ and $j$ are small), as compared to the corresponding deterministic construction of [10], and it also maintains nearly the same accuracy. Our work leaves several open problems - improving the dimensionality reduction bounds for projective clustering, or giving a matching lower bound for the same. Another important open problem is to come up with the dimension reduction step of coreset construction using feature selection algorithms such as row/column subset selection 3 .

\section{References}

1. P. K. Agarwal, S. Har-Peled, and K. R. Varadarajan. Approximating extent measures of points. J. ACM, 51(4):606-635, 2004.

2. P. K. Agarwal, S. Har-Peled, and K. R. Varadarajan. geometric approximation via coresets. Current Trends in Combinatorial and Computational Geometry (E. Welzl, ed.), 2007.

3. C. Boutsidis, M. W. Mahoney, and P. Drineas. An improved approximation algorithm for the column subset selection problem. In Proceedings of the Twentieth Annual ACM-SIAM Symposium on Discrete Algorithms, SODA 2009, New York, NY, USA, January 4-6, 2009, pages 968-977, 2009.

4. K. L. Clarkson and D. P. Woodruff. Low rank approximation and regression in input sparsity time. In Symposium on Theory of Computing Conference, STOC'13, Palo Alto, CA, USA, June 1-4, 2013, pages 81-90, 2013.

5. M. B. Cohen, S. Elder, C. Musco, C. Musco, and M. Persu. Dimensionality reduction for k-means clustering and low rank approximation. In Proceedings of the Forty-Seventh Annual ACM on Symposium on Theory of Computing, STOC 2015, Portland, OR, USA, June 14-17, 2015, pages 163-172, 2015.

6. M. Ester, H. Kriegel, J. Sander, and X. Xu. A density-based algorithm for discovering clusters in large spatial databases with noise. In Proceedings of the Second International Conference on Knowledge Discovery and Data Mining (KDD-96), Portland, Oregon, USA, pages 226-231, 1996.

7. D. Feldman, A. Fiat, and M. Sharir. Coresets forweighted facilities and their applications. In 47th Annual IEEE Symposium on Foundations of Computer Science (FOCS 2006), 21-24 October 2006, Berkeley, California, USA, Proceedings, pages 315-324, 2006.

8. D. Feldman and M. Langberg. A unified framework for approximating and clustering data. In Proceedings of the 43rd ACM Symposium on Theory of Computing, STOC 2011, San Jose, CA, USA, 6-8 June 2011, pages 569-578, 2011.

9. D. Feldman, M. Monemizadeh, C. Sohler, and D. P. Woodruff. Coresets and sketches for high dimensional subspace approximation problems. In Proceedings of the twenty-first annual ACM-SIAM symposium on Discrete Algorithms, pages 630-649, 2010.

10. D. Feldman, M. Schmidt, and C. Sohler. Turning big data into tiny data: Constantsize coresets for k-means, pca and projective clustering. In Proceedings of the Twenty-Fourth Annual ACM-SIAM Symposium on Discrete Algorithms, pages 1434-1453, 2013.

11. S. Har-Peled. No, coreset, no cry. In FSTTCS 2004: Foundations of Software Technology and Theoretical Computer Science, 24th International Conference, Chennai, India, December 16-18, 2004, Proceedings, pages 324-335, 2004.

12. S. Har-Peled and S. Mazumdar. On coresets for k-means and k-median clustering. In Proceedings of the 36th Annual ACM Symposium on Theory of Computing, Chicago, IL, USA, June 13-16, 2004, pages 291-300, 2004. 
13. M. R. Henzinger, P. Raghavan, and S. Rajagopalan. Computing on data streams. In External Memory Algorithms, Proceedings of a DIMACS Workshop, New Brunswick, New Jersey, USA, May 20-22, 1998, pages 107-118, 1998.

14. A. Hinneburg, C. C. Aggarwal, and D. A. Keim. What is the nearest neighbor in high dimensional spaces? In VLDB 2000, Proceedings of 26th International Conference on Very Large Data Bases, September 10-14, 2000, Cairo, Egypt, pages 506-515, 2000.

15. M. W. Mahoney. Randomized algorithms for matrices and data. Foundations and Trends in Machine Learning, 3(2):123-224, 2011.

16. J. M. Phillips. Coresets and sketches. CoRR, abs/1601.00617, 2016.

17. T. Sarlós. Improved approximation algorithms for large matrices via random projections. In 47th Annual IEEE Symposium on Foundations of Computer Science (FOCS 2006), 21-24 October 2006, Berkeley, California, USA, Proceedings, pages 143-152, 2006.

18. K. R. Varadarajan and X. Xiao. A near-linear algorithm for projective clustering integer points. In Proceedings of the Twenty-Third Annual ACM-SIAM Symposium on Discrete Algorithms, SODA 2012, Kyoto, Japan, January 17-19, 2012, pages 1329-1342, 2012.

19. T. Zhang, R. Ramakrishnan, and M. Livny. BIRCH: A new data clustering algorithm and its applications. Data Min. Knowl. Discov., 1(2):141-182, 1997. 


\section{A Appendix}

Proof of Lemma 11\} We first express the term $\|A X\|_{F}^{2}-\|\tilde{A} X\|_{F}^{2}$ in the form of $\operatorname{tr}$ function:

$$
\begin{aligned}
& \|A X\|_{F}^{2}-\|\tilde{A} X\|_{F}^{2}=\operatorname{tr}\left((A X)^{T}(A X)\right)-\operatorname{tr}\left((\tilde{A} X)^{T} \tilde{A} X\right) \\
& =\operatorname{tr}\left(X^{T} A^{T} A X\right)-\operatorname{tr}\left(X^{T} \tilde{A}^{T} \tilde{A} X\right) \\
& =\operatorname{tr}\left(X^{T}\left(A^{T} A-\tilde{A}^{T} \tilde{A}\right) X\right)=\operatorname{tr}\left(X X^{T}\left(A^{T} A-\tilde{A}^{T} \tilde{A}\right)\right) .
\end{aligned}
$$

The above equalities follows due to definition of $\operatorname{tr}$ function $\|A\|_{F}^{2}=\operatorname{tr}\left(A^{T} A\right)$, and due to cyclic and linear properties of tr function (Fact 4). Let we denote the matrix $X X^{T}$ by $P$, and $\left(A^{T} A-\tilde{A}^{T} \tilde{A}\right)$ by matrix $M$. Thus, the problem reduces to bounding the term $\operatorname{tr}(P M)$. Let $\lambda_{i}(M)$ is the $i$ th eigenvalue, and $\left\{w_{i}\right\}_{i=1}^{d}$ be the eigenvectors of $M$, then $M=\Sigma_{i=1}^{d} \lambda_{i}(M) w_{i} w_{i}^{T}$.The following expression holds due to linearity of trace function.

$$
\operatorname{tr}(P M)=\operatorname{tr}\left(P \Sigma_{i=1}^{d} \lambda_{i}(M) w_{i} w_{i}^{T}\right)=\Sigma_{i=1}^{d} \lambda_{i}(M) \operatorname{tr}\left(P w_{i} w_{i}^{T}\right)
$$

Further, we bound the summation $\Sigma_{i=1}^{d} \operatorname{tr}\left(P w_{i} w_{i}^{T}\right)$,

$$
\begin{aligned}
\Sigma_{i=1}^{d} \operatorname{tr}\left(P w_{i} w_{i}^{T}\right) & =\operatorname{tr}\left(P W W^{T}\right)=\operatorname{tr}\left(P^{T} P W W^{T} W W^{T}\right)=\operatorname{tr}\left(P^{T} P W W^{T}\right) \\
& =\operatorname{tr}\left(P W W^{T} P^{T}\right)=\|P W\|_{F}^{2} \leq\|P\|_{F}^{2}=\left\|X X^{T}\right\|_{F}^{2} \leq j
\end{aligned}
$$

where, $W \in \mathbb{R}^{d \times d}$ having columns as eigenvectors of $M$. The above euqalities follow as $P=X X^{T}$, then $P^{T} P=P$; similarly $W W^{T}=W W^{T} W W^{T}$, also $X^{T} X=I, W^{T} W=I$. Finally, the inequality $\|P W\|_{F}^{2} \leq\|P\|_{F}^{2}$ follows from Fact 7

Further, $P=X X^{T}$ has all singular values either 1 or 0 .

$$
0 \leq \operatorname{tr}\left(P w_{i} w_{i}^{T}\right)=w_{i}^{T} P w_{i} \leq\left\|w_{i}\right\|_{2}^{2}\|P\|_{2}^{2} \leq 1
$$

Thus, for $1 \leq i \leq d, \operatorname{tr}\left(P w_{i} w_{i}^{T}\right)$ has $d$ values, and each value is at most 1 (Equation 13), and sum of all of them is at most $j$ (Equation 12).

Further as $M$ is symmetric, we have

$$
\begin{aligned}
M & =A^{T} A-\tilde{A}^{T} \tilde{A}=A^{T} A-(A-(A-\tilde{A}))^{T}(A-(A-\tilde{A})) \\
& =A^{T} A-\left(A^{T}-(A-\tilde{A})^{T}\right)(A-(A-\tilde{A})) \\
& =A^{T} A-A^{T} A+(A-\tilde{A})^{T} A+A^{T}(A-\tilde{A})-(A-\tilde{A})^{T}(A-\tilde{A}) \\
& =(A-\tilde{A})^{T} A+A^{T}(A-\tilde{A})-(A-\tilde{A})^{T}(A-\tilde{A}) \\
& =(A-\tilde{A})^{T}(A-\tilde{A}+\tilde{A})+(A-\tilde{A}+\tilde{A})^{T}(A-\tilde{A})-(A-\tilde{A})^{T}(A-\tilde{A}) \\
& =(A-\tilde{A})^{T}(A-\tilde{A})+(A-\tilde{A})^{T} \tilde{A}+(A-\tilde{A})^{T}(A-\tilde{A})+\tilde{A}^{T}(A-\tilde{A}) \ldots \\
& \ldots-(A-\tilde{A})^{T}(A-\tilde{A}) \\
& =(A-\tilde{A})^{T}(A-\tilde{A})
\end{aligned}
$$


Equality 14 holds due to $(A-\tilde{A})^{T} \tilde{A}=\tilde{A}^{T}(A-\tilde{A})=0$ because rows of $\tilde{A}$ and $(A-\tilde{A})$ lie in orthogonal subspaces. Equality 14 and Fact 5 shows that $M$ is a positive semidefinite matrix, and as a consequence it has all nonnegative eigenvalues. Then, the summation $\sum_{i=1}^{d} \lambda_{i}(M) \operatorname{tr}\left(P w_{i} w_{i}^{T}\right)$ is maximized when $\operatorname{tr}\left(P w_{i} w_{i}^{T}\right)=1$, that is for those eigenvectors which corresponds to largest magnitude eigenvalues of $M$. Thus,

$$
0 \leq \operatorname{tr}(P M)=\Sigma_{i=1}^{d} \lambda_{i}(M) \operatorname{tr}\left(P w_{i} w_{i}^{T}\right) \leq \Sigma_{i=1}^{j} \lambda_{i}(M) .
$$

As a consequence, we have $0 \leq \operatorname{tr}\left(X X^{T}\left(A^{T} A-\tilde{A}^{T} \tilde{A}\right)\right) \leq \Sigma_{i=1}^{j} \lambda_{i}\left(A^{T} A-\tilde{A}^{T} \tilde{A}\right)=$ $\Sigma_{i=1}^{j} \sigma_{i}^{2}(A-\tilde{A})=\left\|(A-\tilde{A})_{j}\right\|_{F}^{2}$. Here matrix $(A-\tilde{A})_{j}$ is the matrix restricted to rank $j$ of the matrix $A-\tilde{A}$. Further, as matrix $\tilde{A}$ is of rank at most $m$, $\tilde{A}+(A-\tilde{A})_{j}$ is of rank at most $m+j$. Thus, we have

$$
\begin{aligned}
\left\|A-\left(\tilde{A}+(A-\tilde{A})_{j}\right)\right\|_{F}^{2} & \geq \Sigma_{i=m+j+1}^{d} \sigma_{i}^{2} \\
\|A-\tilde{A}\|_{F}^{2}-\left\|(A-\tilde{A})_{j}\right\|_{F}^{2} & \geq \Sigma_{i=m+j+1}^{d} \sigma_{i}^{2} \\
\left\|(A-\tilde{A})_{j}\right\|_{F}^{2} & \leq\|A-\tilde{A}\|_{F}^{2}-\Sigma_{i=m+j+1}^{d} \sigma_{i}^{2} \\
\left\|(A-\tilde{A})_{j}\right\|_{F}^{2} & \leq(1+\epsilon)\left\|A-A^{(m)}\right\|_{F}^{2}-\Sigma_{i=m+j+1}^{d} \sigma_{i}^{2} \\
& =(1+\epsilon) \sum_{i=m+1}^{d} \sigma_{i}^{2}-\Sigma_{i=m+j+1}^{d} \sigma_{i}^{2} \\
& =\Sigma_{i=m+1}^{m+j} \sigma_{i}^{2}+\epsilon \sum_{i=m+1}^{d} \sigma_{i}^{2} \\
& \leq \epsilon \Sigma_{j+1}^{d} \sigma_{i}^{2}+\epsilon \sum_{i=j+1}^{d} \sigma_{i}^{2} \\
& =2 \epsilon\left\|A X^{\perp}\right\|_{F}^{2}
\end{aligned}
$$

Inequality [15] follows from Fact 1 Inequality 16 follows from Fact 3 where $(A-$ $\tilde{A})_{j}=(A-\tilde{A}) X X^{T}$ for an orthonormal matrix $X \in \mathbb{R}^{d \times j}$; Inequality 17 follows from Theorem 2 Inequality 18 holds as $m=\left\lceil\frac{j}{\epsilon}\right\rceil$, and due to the following:

$$
\Sigma_{i=m+1}^{m+j} \sigma_{i}^{2} \leq j \sigma_{m+1}^{2}=\epsilon m \sigma_{m+1}^{2} \leq \epsilon m \sigma_{j+1}^{2} \leq \epsilon \sum_{i=j+1}^{m+j} \sigma_{i}^{2} \leq \epsilon \Sigma_{i=j+1}^{d} \sigma_{i}^{2} .
$$

\section{Proof of Lemma 12}

$$
\begin{aligned}
\left\|A^{*} X^{*} X^{* T}-A X^{*} X^{* T}\right\|_{F}^{2} & =\left\|A X^{*} X^{* T}-A^{*} X^{*} X^{* T}\right\|_{F}^{2} \\
& =\left\|\left(A-A^{*}\right) X^{*} X^{* T}\right\|_{F}^{2} \\
& =\operatorname{tr}\left(\left(A-A^{*}\right) X^{*} X^{* T}\left(\left(A-A^{*}\right) X^{*} X^{* T}\right)^{T}\right) \\
& =\operatorname{tr}\left(\left(A-A^{*}\right) X^{*} X^{* T} X^{*} X^{* T}\left(A-A^{*}\right)^{T}\right) \\
& =\operatorname{tr}\left(\left(A-A^{*}\right)^{T}\left(A-A^{*}\right) X^{*} X^{* T}\right) \\
& =\operatorname{tr}\left(X^{*} X^{* T}\left(A^{T} A-A^{* T} A^{*}\right)\right) \\
& \leq \frac{\epsilon^{2}}{26}\left\|A X^{* \perp}\right\|_{F}^{2}
\end{aligned}
$$


Equality 19 holds due to cyclic property of tr function and $X^{* T} X^{*}=I$; Equality 20 holds due to cyclic property of tr, and $\left(A-A^{*}\right)^{T}\left(A-A^{*}\right)=(A-$ $\left.A R^{*} R^{* T}\right)^{T}\left(A-A R^{*} R^{* T}\right)=A^{T} A-A^{* T} A^{*}$ (after simplification, similar to Equation 14); finally Inequality 21 holds by following the steps of proof of Lemma 11 (from Equation 11), where we replace $\epsilon$ by $\frac{\epsilon^{2}}{52} ; j$ by $j^{*}, A$ by $A^{*}, X$ by $X^{*}$. 